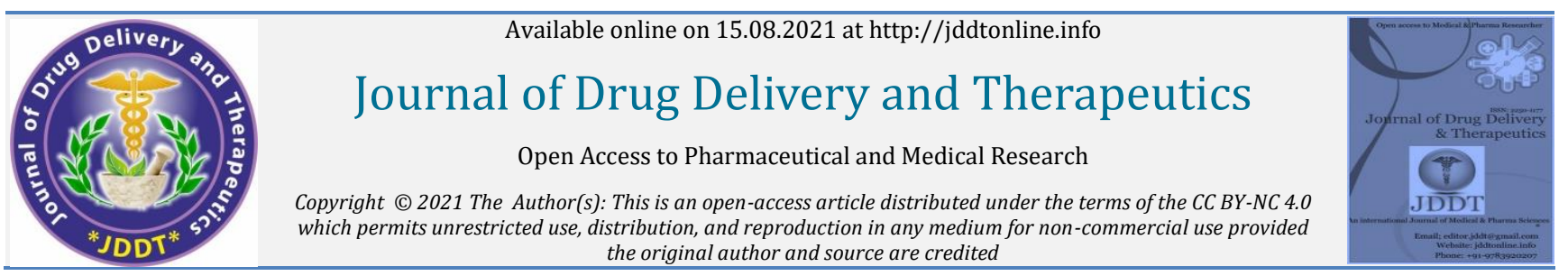

Open Access Full Text Article the original author and source are credited

Review Article

\title{
The Relevance of Defined Daily Dose concept in Drug Utilisation Research: A Review
}

\author{
Agnus Baiju*, Rosmin Jacob, K. Krishnakumar \\ Department of Pharmacy Practice, St James College of Pharmaceutical sciences, St. James Hospital Trust Pharmaceutical Research Center (DSIR \\ Recognised), Chalakudy, Kerala, India
}

\section{Article Info:}

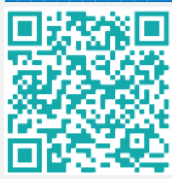

\section{Article History:}

Received 07 June 2021

Reviewed 11 July 2021

Accepted 20 July 2021

Published 15 August 2021

\section{Cite this article as:}

Baiju A, Jacob R, Krishnakumar K, The Relevance of Defined Daily Dose concept in Drug Utilisation Research: A Review, Journal of Drug Delivery and Therapeutics. 2021; 11(4-S):162-165 DOI: http://dx.doi.org/10.22270/jddt.v11i4-S.4897

\section{Abstract}

According to World Health Organisation (WHO), drug utilisation is defined as the marketing distribution, prescription and the use of drugs in society with special focus on resulting medical, social and economic consequences and it has turned into a powerful scientific too for ensuring the rational and cost-effective use of drugs. A standard method is required to identify and aggregate drug data. This manuscript provides an overview of the WHO Anatomical Therapeutic Chemical (ATC) classification and the associated measure, Defined Daily Dose (DDD), as a methodology for evaluating drug utilisation.

Keywords: Drug utilisation research, Anatomical Therapeutic Chemical classification system Defined Daily Dose, Rational drug use.

*Address for Correspondence:

Agnus Baiju, Department of Pharmacy Practice, St James College of Pharmaceutical sciences, St. James Hospital Trust Pharmaceutical Research Center (DSIR Recognised), Chalakudy, Kerala, India

\section{Introduction:}

The emergence of drug utilization as a research area facilitated to study drug prescribing and drug usage in a scientific and formal manner 1 . Due to huge drug consumption, the rational drug use has a strong impact on ensuring drug safety 2 .

In 1985, the World Health Organization (WHO) organized a conference to promote the rational use of drugs. According to the WHO, the rational use of drugs requires that patients receive medications appropriate to their clinical needs, in doses that meet their own individual requirements for an adequate period of time, and at the lowest cost to them and their community ${ }^{3}$.

Drug utilisation research (DUR) is an essential research approach in health services research to have a clear idea of drug use pattern, to identify early signs of irrational drug use and to enhance the quality of drug use 2. Hence, drug utilisation must be followed up systematically and comprehensively after the drugs have been marketed ${ }^{4}$.

In 1981, the WHO adopted the ATC/DDD system for DUR, which is so-called as Anatomical Therapeutic Chemical (ATC) classification and Defined Daily Dose (DDD). The abbreviation DDD in block capital is generally described as the WHO value.

Mostly DURs are carried out at hospital settings where the data are collected through inpatient and outpatient department. At present, DUR is a common technique used to assess drug use, and defined daily dose is a popular concept adopted in most DURs ${ }^{2}$.

\section{Anatomical and Therapeutic Chemical Classification (ATC) System:}

The Anatomic Therapeutic Chemical classification system is generally used in conjunction with the DDD method. It provides global standard for classifying drugs ${ }^{11}$. The ATC/DDD system was initially developed as an aid for presenting drug consumption figures. It was originally developed by the Norwegian Medicinal Depot, which became a WHO Collaborating Centre for Drug Statistics Methodology 5

\section{WHO ATC Classification}

The ATC system managed by the WHO Collaborating Centre for Drug Statistics Methodology (WHOCC) is responsible for classifying and arranging the drugs into comparable categories. To carry out these, the system makes use of information about the organ and system on which a drug acts, as well as the drug's chemical, pharmacological and therapeutic properties ${ }^{6}$.

The ATC system, if employed universally, could facilitate international comparisons for drug consumption studies 1 . The ATC/DDD system can also provide a basis for the evaluation of long-term trends in drug use 5 . 
Table 1: WHO ATC Classification ${ }^{5}$

\begin{tabular}{|l|l|}
\hline ATC Group & Affected system \\
\hline A & Alimentary tract and metabolism \\
\hline B & Blood and blood-forming organs \\
\hline C & Cardiovascular system \\
\hline D & Dermatological \\
\hline G & Genito-urinary system and sex hormones \\
\hline H & Hormonal preparations, excluding sex hormones and insulins \\
\hline J & Anti-infective for systemic use \\
\hline L & Antineoplastic and immunomodulating agents \\
\hline M & Musculoskeletal system \\
\hline N & Nervous system \\
\hline P & Antiparasitic, insecticides and repellents \\
\hline R & Respiratory system \\
\hline S & Sensory organs \\
\hline V & Various \\
\hline
\end{tabular}

\section{How ATC Works}

As stated in the ATC classification system mentioned in Table 1 , drugs are classified into 14 main groups. The ATC system consists of five hierarchical levels: a main anatomical group, two therapeutic subgroups, a chemical-therapeutic subgroup, and a chemical substance subgroup 5 .
Drugs reviewed by the Centre receive an ATC code based on this five-tiered system. The example given in Table 2 illustrates the ATC code for metformin, a medicament used to treat type 2 diabetes ${ }^{6}$.

Medicinal products are classified based on the main therapeutic indication for the principal active ingredient 5 Each pharmaceutical preparation has just one ATC code ${ }^{1}$

Table 2: ATC code for metformin ${ }^{6}$

\begin{tabular}{|l|l|}
\hline & ATC Schematic Structure \\
\hline Code & Specification \\
\hline A & Alimentary tract and metabolism (1st level, anatomical main group) \\
\hline A10 & Drugs used in diabetes (2nd level, therapeutic subgroup) \\
\hline A10B & Blood glucose lowering drugs, excl. insulins (3rd level, pharmacological subgroup) \\
\hline A10B A & Biguanides (4th level, chemical subgroup) \\
\hline A10B A02 & Metformin (5th level, chemical substance) \\
\hline
\end{tabular}

However, some active medicinal substances may have more than one ATC code, if the drug has different uses at different strengths (acetylsalicylic acid as a platelet aggregation inhibitor in addition to analgesic-antipyretic), dosage forms (timolol to treat hypertension and to treat glaucoma), or both (medroxyprogesterone for cancer therapy and as a sex hormone). Prednisolone is an example of a medicament that has six different ATC codes ${ }^{5}$.

Fixed- dose combination products pose classification difficulties, thus categorised by principle use ${ }^{1}$. For example, a combination product that contains an analgesic and a tranquilizer is classified as an analgesic, despite it also contains a psychotropic substance 5 .

\section{Defined Daily Dose (DDD):}

In order to overcome the limitations of expressing consumption concerning costs or units prescribed or sold, another measurement unit was confirmed and has come into widespread use, namely the Defined Daily Dose (DDD). A DDD is defined for each drug (particularly, each active ingredient) 1 .

\section{Definition of DDD}

The unit of measure created to support the WHO ATC classification system is the DDD. The DDD is a technical unit of measurement assigned for different drug formulations ${ }^{12}$. It is defined as the "assumed average maintenance dose per day for a drug used for its main indication in adults"7.

The majority of drugs are available in different dosages for different symptoms and this must be considered while evaluating drug consumption figures. The DDD have two assumptions based on which it is used, namely that patients take the medication (that is, that patients are compliant); and 
Baiju et al

the doses used for the major indication are the average maintenance doses ${ }^{1}$.

Where possible, the DDD is indicated with reference to the weight of active ingredients using the most appropriate units, for example, g (gram), mg (milligram), ug (microgram), mmol (millimole), E (unit), TE (thousand units) or ME (million units). For practical reasons, the DDD is formulated on use in adults and is rarely used in children ${ }^{1}$.

\section{How DDD Works}

The defined daily dose is an artificially and arbitrarily created statistical measurement used in research purposes to compare the drugs prescribed and to evaluate the effects of interactions on prescribing pattern ${ }^{12}$. Only drugs with an ATC code can have DDD's ${ }^{6}$. Each DDD links to a specific ATC code and provides common dosage allowance for each drug under one administration route 2 . The DDD does not necessarily correspond to the recommended or the prescribed daily dose (PDD) ${ }^{10}$.

The value of DDD is decided by the WHO International Working Group for Drug Statistics Methodology consisting of 12 expert members selected by WHO Headquarters 10. Further details may be accessed from WHO Guidelines for ATC classification and DDD assignment and the Web site of the WHOCC ${ }^{2}$.

The DDD method has been modified in situations like;

- On the basis of administration method, drugs are available in different dosages, hence one DDD could be used for the oral route and another for the parenteral route.

- In case of fixed combinations, where the defined dose cannot be expressed in weight of active ingredient, it is expressed as the number of single doses (such as the number of tablets, capsules or suppositories) normally used per day to obtain the desired therapeutic effect 1 .

Due to new indications or newer research, dosages may change over time. Hence with regular revisions, researchers must determine DDD and carefully document it, such that the resulting drug statistics may be adequately interpreted 5 . Numerous modifications in DDDs are inconvenient for longterm studies on drug utilization 1.

Consumption in hospitals is calculated just the same as the general population and is typically expressed as the number of DDD per 100 bed days. In making the calculation, the days of admission and discharge are usually counted together as one bed day 1.

Although this system has clear limitations, it has been employed very successfully for its prime purpose, namely research into drug utilization ${ }^{6}$.

\section{Applications of DDD}

DURs are normally done in hospital or ambulatory care settings. Limited studies are carried out in community pharmacy due to the difficulty in generating the necessary data in this setting 1 . Ultimately, the DDD results in the numerical identification of the quantity of drug product consumed per day, per 1000 residents 6 .

The DDD have been employed in research purposes that one may:

- Measure the intensity of intake of a specific drug product in a single nation or between nations overtime. Thus, national publications give health care professionals an outline of drug consumption in the country ${ }^{6}$.
Journal of Drug Delivery \& Therapeutics. 2021; 11(4-S):162-165

- Make international comparisons;

- Evaluate the effect of educational programs directed either at the prescriber or the patient;

- Document the relative therapy intensity with various drug class;

- To enhance drug usage and to follow changes in drug class 6 ; and

- Assess regulatory effects on prescribing patterns ${ }^{1}$.

Advantages and Limitations of using the WHO ATC/DDD methodology:

\section{Advantages}

- Drug classes can be compared between or within regions or countries and between time periods. For example, the DDDs per 1000 inhabitants per day unit had been employed to demonstrate national and international differences in the utilisation of antidiabetics, antibiotics and cardiovascular drugs. It has aided in measuring the frequency of disease and its consequences 8 .

- ATC and DDDs are maintained by an international organisation, updated annually, and declared as a userfriendly index (in print and online).

- Useful for working with readily available gross drug statistics at various levels of the health system, e.g. sales data, pharmacy claims data, patient encounter-based data.

- Drug utilisation statistics could determine overuse, underuse or misuse of drugs 6 .

- Relatively easy and inexpensive to use.

- DUR can be utilised to improve medical care and costcontainment, and are useful for measuring or comparing the economic impact in the population ${ }^{9}$.

- ATC codes are alphanumeric and categorical, and their use is intuitive for the most part.

- Specific medicinal substances can be identified by the fifth level ATC code

- ATC codes may be incorporated into administrative databases and statistical analysis packages as 'groupers' for Drug Identification Numbers and NDCs belonging to specific drug classes

- Useful to aggregate volume measures of drug claims within and across drug classes without the same limitations of simple volume measures such as number of tablets and number of prescriptions ${ }^{7}$.

\section{Limitations}

Although the establishment of DDD has meant a great progress in the measurement of drug consumption, a few problems and limitations exist, namely:

- Specific medicinal substances may have multiple DDD.

- The DDD methodology is calculated on the basis of the units issued or sold. The DDD is not necessarily similar to the average doses actually prescribed nor yet to the mean dose actually ingested every day. The doses prescribed and taken in a particular community will alter with the indications actually predominating, national or regional therapeutic traditions and with the attitude of patients ${ }^{1}$.

- An ATC code may be retired over time. 
- Different versions of the ATC/DDD exist and DDDs can change with time; historical documentation is required for longitudinal analysis.

- Does not imply judgment of efficacy or allow price comparison.

- Not appropriate for cost of illness, cost-benefit, costeffectiveness, and cost-utility analyses as DDD comparison may not be based on adequate data regarding equivalency of surrogate or final outcomes.

- It is fundamental to consider, and adapt if necessary, the size of the population used as a denominator. Usually general consumption is calculated for the total population (all age groups), but drug use is often concentrated within certain specific category (such as, oral contraceptives and some vaccines). In certain cases, it might be more meaningful to take such a group as a denominator. The consumption, for example, of oral contraceptives is routinely given as the proportion of women 18 to 44 years old using these preparations 1 .

- Some combination products are assigned a fixed DDD (Unit Dose-UD) and may be assigned an ATC separate from additional active ingredients 7 .

\section{Conclusion:}

Drug utilization studies is widely used research tool and have an increasing significance in pharmacoepidemiology with the aid of linking more closely to other sectors like public health, pharmacovigilance, pharmacoeconomics and pharmacology. The information collected can be employed to conduct screening of patients who are at higher risk for drug-induced illnesses due to concomitant therapies, abuse or overuse of drugs ${ }^{9}$.

DURs can be done in hospital setting since data can be retrieved from inpatient or outpatient department. This research is not usually seen in other settings ${ }^{2}$. Several national and international comparative studies are conducted at the population level under the guidance of WHO Collaborating Centre for Drugs Statistics Methodology. It is usually represented as number of DDDs per 1000 inhabitants per day. Even so, DDD is used at the individual level ${ }^{8}$.

Methodologies used to estimate drug utilisation are inconsistent, as the definition of DDD varied by using unauthorised sources or sources based on personal experiences. Future high quality DURs with consistent methodology are needed for DURs conducted to reach an international capacity ${ }^{2}$.

A dose standard unit which is recognised worldwide is essential in drug utilisation studies, specifically if different
Journal of Drug Delivery \& Therapeutics. 2021; 11(4-S):162-165 studies are to be compared. DDD was highly beneficial and advantageous than other alternatives. Therefore, there is a rationale to use DDD as the sole standard dose unit in all pharmacoepidemiologic studies ${ }^{8}$. Drug utilization studies using ATC/DDD system need to be promoted and carried out on an ongoing basis. DUR is important for rational use of drugs ${ }^{11}$.

\section{References:}

1. Trute I, A Review of Drug Utilization Studies and Methodologies, Jordan Journal of Pharmaceutical Sciences, 2008; 1(2):91-103.

2. Teng $L$ et al., Review of the use of defined daily dose concept in drug utilisation research in China, Pharmacoepidemiology and Drug Safety, 2012; 21(10):1118-1124. https://doi.org/10.1002/pds.3240

3. Atif M, Scahill S, Muhammad Azeem, Muhammad Rehan Sarwar, Zaheer-Ud-Din Babar, Drug utilization patterns in the global context: a systematic review, Health Policy and Technology, 2017; 6(4):457-470. https://doi.org/10.1016/j.hlpt.2017.11.001

4. The Selection of Essential Drugs: Report of a WHO Expert Committee. Technical Report Series no.615. Geneva: World Health Organization, 1977.

5. Wettermark B et al., Studies of Drug Utilization, 373-410. https://doi.org/10.1002/9781118949740.ch1

6. Wertheimer AI, Santella TM, Problems Using the Defined Daily Dose (DDD) as a Statistical Basis for Drug Pricing and Reimbursement, 1-15.

7. Sketris et al., The Use of the World Health Organisation Anatomical Therapeutic Chemical/Defined Daily Dose Methodology in Canada, Drug Information Journal, 2004; 38(1):15-27. https://doi.org/10.1177/009286150403800104

8. Merlo J, Wessling A, Melander A, Comparison of dose standard units for drug utilisation studies, Eur J Clin Pharmacol, 1996; 50(1-2):27-30. https://doi.org/10.1007/s002280050064

9. Gama H, Drug Utilization Studies, Arquivos de medicina, 2008; 22(2/3):69-74.

10. WHO Collaborating Centre for Drug Statistics Methodology. ATC/DDD Index. 2020 Available from https://www.whocc.no/atc_ddd_index/

11. Bachhav SS, Kshirsagar NA, Systematic review of drug utilization studies \& the use of the drug classification system in the WHOSEARO Region, Indian J Med Res, 2015; 142(2):120-129. https://doi.org/10.4103/0971-5916.164223

12. Kumar A et.al., Point prevalence study of use and consumption of antibiotics by using Anatomical and Therapeutic Chemical Classification and Defined Daily Dose Methodology in Tertiary Care Hospital, Indian Journal of Pharmacy Practice, 2020; 13(2):151-155. https://doi.org/10.5530/ijopp.13.2.23 